\title{
Wireless network of autonomous environmental sensors
}

\author{
Tom Torfs, Chris Van Hoof, Steven Sanders, Christophe Winters, Steven Brebels
}

IMEC, Leuven, Belgium, \{torfst,vanhoofc,sanders, winters,brebels \} @imec.be

\begin{abstract}
A wireless network based on macro-scale sensor modules (originally $45 \times 35 \times 30 \mathrm{~mm}^{3}$, currently $14 \times 14 \times 18 \mathrm{~mm}^{3}$ with integrated coplanar antenna) has been demonstrated. The modules can extract their energy from the environment (e.g. with solar cells), and detect various environmental parameters. The network can be configured as a multi-hop "web" of sensors which builds itself automatically according to configurable optimization parameters, or as a "star" topology, where each sensor module transmits information directly to the base station. Average power consumption for each complete module is approx. $100 \mu \mathrm{W}$. This ability for drastic miniaturization and the simultaneous addition of power autonomy while maintaining modularity and reusability contrast to existing work where wireless environmental sensor systems were at least one order of magnitude larger in volume or consisted of applicationspecific systems-on-a-chip.
\end{abstract}

\section{INTRODUCTION}

Wireless networks of tiny, intelligent sensors offer useful applications in environmental sensing, intelligent offices or houses, security, health and comfort applications around and even inside the body[1][2]. A macro-scale environmental sensor network serves as a useful application of the technology and allows exploring the technological challenges of further integration, minimizing power consumption and improving the wireless communication. With the successful implementation of this macro-scale network, it is possible to examine network topologies and power consumption in real-world situations. Based on these results, further integration and further power consumption reduction can be achieved, leading towards tiny, autonomous sensors required for body area applications[9].

\section{SENSOR MODULE DESIGN}

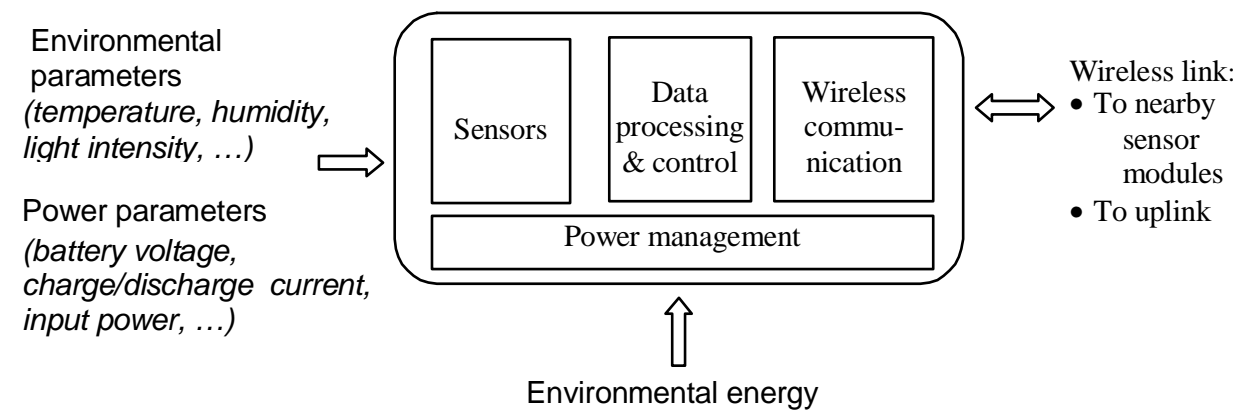

Figure 1: Block schematic of autonomous wireless sensor module

Figure 1: Block schematic of autonomous wireless sensor module

\section{Architecture}

A sensor module consists of several functional blocks as seen in Figure 1. Each block has a clearly delineated task in the sensor module's operation. The specific requirements for a particular block's tasks can differ depending on the application:

- A large-area low-density sensor network deployment will require a more powerful radio than a short range indoor or on-the-body sensor application.

- Applications where high data rates and complex signal processing functions are required will benefit from a more powerful digital signal processor.

- Sensors and associated sensor electronics will vary from application to application.

- For some applications the power can be provided by an appropriate primary battery, whereas others call for a complete power management system that can scavenge energy from the environment, properly condition this energy, and use it to keep a secondary battery charged.

Therefore if a wireless sensor module architecture is to be suitable for a broad range of applications, it should be designed in a modular fashion. The blocks in Figure 1 have been implemented as distinct hardware layers which can be plugged together like Lego blocks to obtain a sensor module with the desired functionality. The data processing \& control block can be reprogrammed in-system to provide this application-tailored functionality.

\section{Hardware}

Each functional block has been implemented as a $14 \times 14$ $\mathrm{mm}^{2}$ printed circuit board. The boards are stacked vertically with compact connectors on the four corners, which provide both the electrical and first-order mechanical interconnections (see Figure 2).

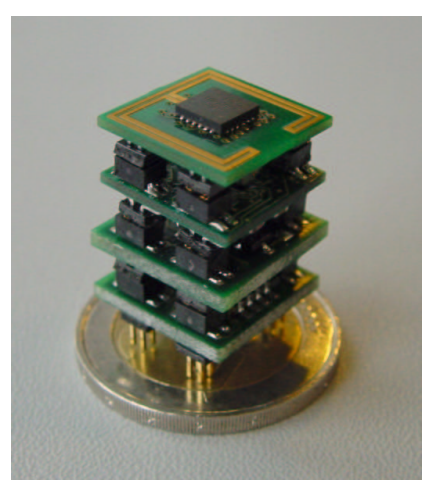

Figure 2: Sensor module on 2-euro coin 
The top layer is responsible for the wireless communication. It consists of a single-chip short-range $2.4 \mathrm{GHz}$ transceiver (nRF2401, 18nJ/bit) to which we added a custom coplanar integrated folded dipole antenna, designed for a system-ina-cube[9].

The next layer implements data processing and control of the entire module. An MSP430 microcontroller was selected for its low active power (0.6nJ/instruction), low standby power $(2 \mu \mathrm{W})$, fast wakeup from standby to active mode $(6 \mu \mathrm{s})$ and on-chip 12-bit analog-to-digital converter. To meet the size requirements imposed by the compact sensor module design, a bare-die component is used, wirebonded directly to the printed circuit board, and encapsulated by a glob-top coating. A $32.768 \mathrm{kHz} \pm 20 \mathrm{ppm}$ crystal provides the system's local time reference. The controller's software is programmed in-system over lines that are multiplexed with normal I/O functionality. The connectors give access to 16 signals, 12 of which are general purpose I/O's (including 4 analog-capable inputs).

The combination of the control and the wireless layer, designed to work closely together, form the core of the wireless module. Additional layers fill in the sensing/actuating and power management functionality.

A power management layer has been implemented which accepts power from an energy scavenger such as a compact solar cell or thermo-electric generator, conditions it to a suitable DC voltage, and uses it for keeping a secondary battery charged. The overall conversion efficiency of the power management circuitry varies from 60 to $70 \%$ when operating in the target power range of 100 to $200 \mu \mathrm{W}$. More efficient power conversion circuitry is under development, but care needs to be taken at these power levels that the static power consumption remains sufficiently low in order not to completely offset the gain in efficiency.

A final component in the sensor module is a sensor layer, which presently measures environmental parameters such as temperature $\left( \pm 0.4^{\circ} \mathrm{C}\right)$, relative humidity $( \pm 2 \%)$ and illumination $( \pm 3 \%$, lux $)$. These sensors were specifically selected for low active power consumption and very low standby power consumption. Other sensors layers can be added or substituted. When many sensors have to communicate with the controller, standard sensor communication interfaces such as Serial Peripheral Interface (SPI) or Inter Integrated Circuit $\left(\mathrm{I}^{2} \mathrm{C}\right)$ allow sharing a single communication bus. The choice of sensors will naturally impact on the power budget. Sensors that can be duty-cycled with a very low standby current and that can operate from a low battery supply voltage $(2 . .3 .6 \mathrm{~V})$ without the need for intermediate DC/DC conversion, will result in a lower-power sensor module (which translates to more autonomy and/or smaller energy scavengers and battery).

\section{Battery}

Even in the presence of a near-constant power source, an energy buffer will be needed to provide the peak currents for the radio transmitter and receiver. Supercapacitors are generally not suitable because their low energy density and linearly decaying voltage prohibit sustained peak currents (e.g. to acquire synchronization). For the most commonly used energy scavenger, the solar cell, much larger energy buffers are needed in most applications, to overcome periods with absence of light.

It is therefore clear that a battery is needed in the sensor module system. Depending on the application a primary battery, with no need for a specific power management layer or energy scavenger, can suffice. In applications where an energy scavenger is employed a secondary battery is appropriate.

The prototype sensor module uses a 2-cell NiMH battery for a nominal voltage of $2.4 \mathrm{~V}$. NiMH was chosen because of its relative ease of charging, safety, environmental friendliness, large peak current capability and lack of severe memory effect[5]. The large self-discharge (2-3\% per day) is not a problem in an energy scavenging application. A custom prismatic Li-Ion battery with higher energy density is in development for longer autonomy and higher-power applications. This requires a modified power management layer and the addition of safety circuitry.

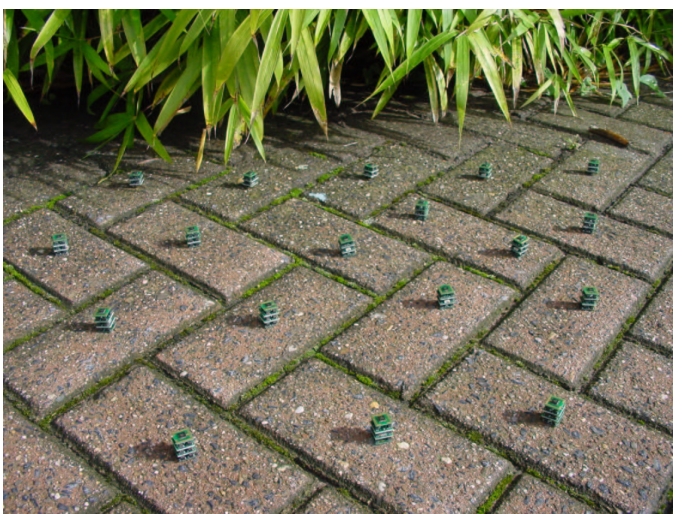

Figure 3: small-scale sensor network

\section{NETWORK DESIGN}

\section{Network architecture}

Two topologies are supported for the sensor network. The first topology is the star topology (Figure 4), where all modules communicate directly with a 'base station'.

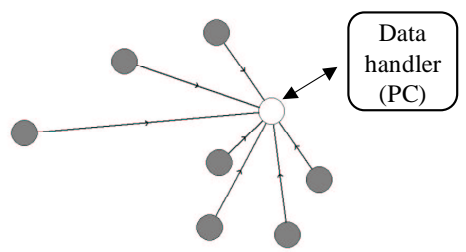

Figure 4: star topology sensor network

The base station requests and aggregates the sensor data, performs additional data processing if needed, and transmits the data to the PC (over USB), where it can be visualized, 
stored and analyzed by application-specific software. Commands, parameters and settings go the other way, with the base station relaying the commands it receives from the PC to a specific sensor module or as a broadcast to the entire network.

This type of network is the most suitable for short-range applications such as indoor or on-the-body measurements, where the base station can be connected to something like a Personal Digital Assistant (PDA). In such a situation, where all modules are well within radio range of the base station, the single-hop approach for communicating between each sensor module and the base station will result in the lowest overall power consumption[3].

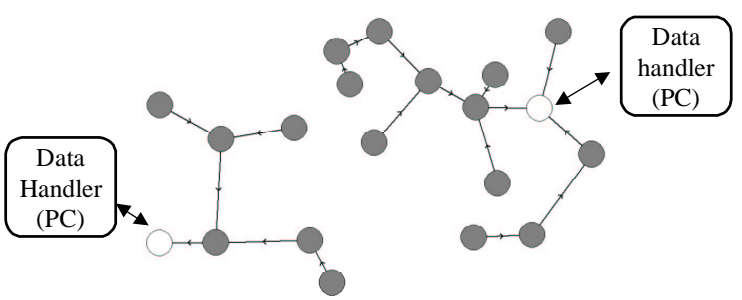

Figure 5: web topology sensor network

For larger-scale networks the star approach can become infeasible because of the radio power that would be required for direct communication between distant sensor modules and the base station. For these applications a web topology is a possible solution[4]. Modules communicate only with their direct neighbors, which can be reached with the lowpower radio. These neighbors relay the data in turn to their neighbors, etc., until the data reaches the base station. The reverse process is used for transmitting commands, parameters and settings to individual modules or the entire network.

The web topology (Figure 5) allows redundancy by routing around dysfunctional modules. Where data loss is critical, this redundancy can be implemented by letting each data packet follow multiple paths. However, this comes at the price of increased overall power consumption. In the web topology as implemented, each module in the network selects a single downlink which receives its data. If the downlink is detected as dysfunctional by the uplink module or one of the modules further downstream (because of repeated silence/data loss), the uplink module will seek a new downlink. This way, a dysfunctional module can only cause significant data loss for a few measurements, after which it will be removed from the network hierarchy and only its own data will be lost. When it becomes functional again (e.g. an empty battery gets charged when energy becomes available), it will request to be taken up into the network, and will start transmitting data again. The same method allows adding new modules to the network.

A simulation program was written to model larger scale networks and assess the performance of network build-up algorithms for creating the web topology. The result of these simulations were verified in small-scale networks of 5 to 20 modules.

\section{Media access control (MAC)}

The modules in the network share a single communication medium, a radio channel in the $2.4 \mathrm{GHz}$ band. The low-duty cycle, non time-critical measurements typical for a network of low-power sensor modules, allow for a time division multiple access (TDMA) method to share the medium. This method works fine for small to medium scale networks, but the most straightforward implementation does not scale up well to very large networks. For larger networks it is possible to re-use TDMA timeslots in distant parts of the network; alternatively frequency division multiplexing can be added. Including this functionality requires relatively small adaptations to the controller software.

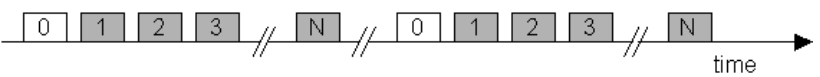

Figure 6: basic TDMA cycle

The basic TDMA cycle is shown in Figure 6. Each module has a unique address. The addresses can be either assigned statically, or dynamically during the build-up of the network. The address translates to a timeslot. The base station, with address 0 , transmits a frame (empty or containing commands) at time $\mathrm{t}=0$. The other modules receive this frame, synchronize their local clocks to it, and store any commands for processing or forwarding. Then they wait for the timeslot corresponding to their address and transmit data during this timeslot. This cycle repeats at specific, relatively long, time intervals.

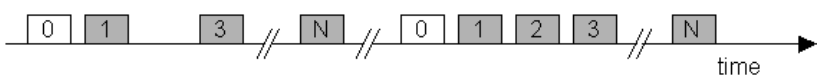

Figure 7: multi-hop TDMA cycle

For multi-hop networks (web topology), some modules will not be able to synchronize on the base station directly. They will instead synchronize on their downlink. An example is shown in Figure 7. Module 2 synchronizes on its downlink 3 instead of base station 0 , therefore it takes an extra TDMA cycle before module 2 is taken up in the network. In a multi-hop network the modules have uplinks as well as a downlink, so the receiver has to be turned on not only during the downlink's timeslot but also during the timeslots of the module's uplinks.

When a module is inserted into the network or becomes functional again after a prolonged downtime, its potential downlinks will not have their receivers enabled during the module's timeslot. As shown in Figure 8, an access timeslot is reserved during which all receivers are periodically enabled to listen for potential new modules on the network.

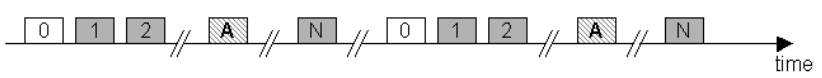

Figure 8: TDMA cycle with access slot 
For the multi-hop web topology a MAC protocol is needed to allow bidirectional communication throughout the network. The synchronization methods are designed in a robust manner to deal with lost frames without losing synchronization, and to re-acquire synchronization intelligently should it be lost. However, rare events such as long series of lost communication frames, failing or restarting modules, will cause a resynchronization, which can increase the power consumption (see Figure 10). In a star network with unidirectional data transmission a power-saving alternative is available: no MAC protocol as such. The modules transmit their data at specific intervals, counting on the low duty cycles of the sensors to avoid collisions. Random or deterministic shifts in the access times to the medium are required to avoid prolonged collision problems caused by slow relative oscillator drift.

\section{POWER CONSUMPTION RESULTS}

A sensor module's power consumption profile during a TDMA cycle is shown in Figure 9.

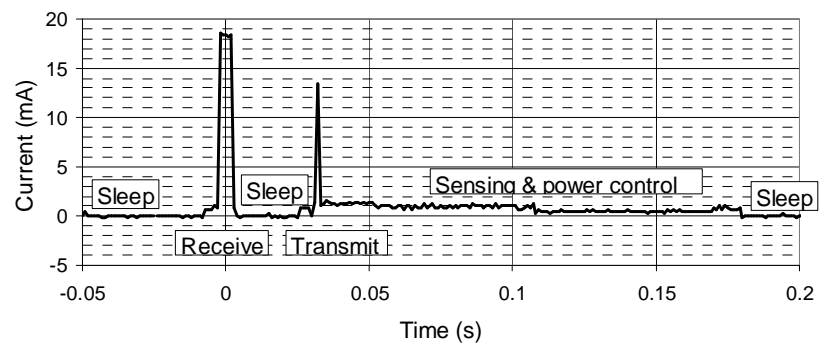

Figure 9: Power consumption profile (no uplinks)

This TDMA cycle returns at a specified measurement interval, with the system returning to a $6 \mu \mathrm{W}$ sleep mode in between. The resulting average power consumption for long measurement intervals is plotted in Figure 10. For unsynchronised transmission in a star network and ideal (errorless) synchronized star or multi-hop networks the average power converges to the sleep power. In the latter case frame errors and synchronization loss introduce extra power consumption which limits further power reduction by using longer intervals. For intervals longer than 5 minutes the synchronization model has to be modified and the curve cannot be simply extrapolated further.

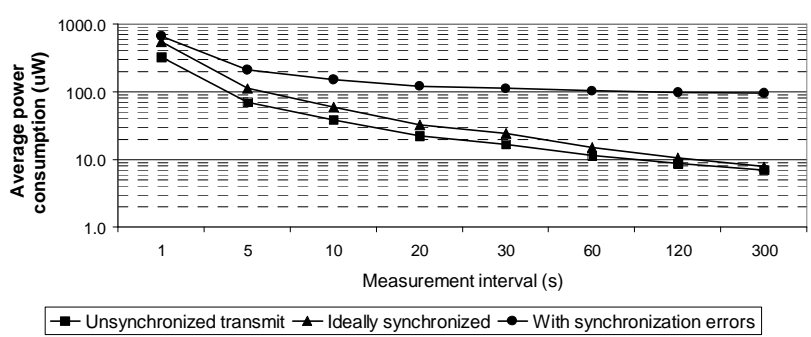

Figure 10: Power in function of measurement interval
For realistic measurement intervals of 30 seconds to 5 minutes, and accounting for synchronization errors, the average power consumption is around $100 \mu \mathrm{W}$. In order to provide this power with a solar cell, including the power conditioning losses and the daylight/night ratio, a solar cell of $1 \mathrm{~cm}^{2}$ in combination with a $1 \mathrm{mAh}$ buffer battery to overcome the nights will suffice for outdoor use. Alternatively, 2xAAA alkaline batteries will result in a self-discharge limited lifetime of 2 to 3 operational years, and a compact $1 / 3 \mathrm{~N}$ $\mathrm{Li} / \mathrm{MnO}_{2}$ battery will allow 4 months of continuous operation.

\section{CONCLUSIONS}

A functional network of miniaturized sensor modules with power autonomy was created, while maintaining modularity and flexibility. This contrasts to existing work where wireless environmental sensor systems were at least one order of magnitude larger in volume[5][6] or consisted of application-specific systems-on-a-chip[7][8].

\section{ACKNOWLEDGMENTS}

Bert Gyselinckx, Walter De Raedt and Tomas Webers from IMEC's HUMAN++ team contributed to the hardware design of the miniature sensor module[9].

Thesis students Wim Tormans, Davy Janssen, Dieter Schepens, Pieter De Smet, Broos Fonck contributed to the development of the macro-scale sensor module network.

\section{REFERENCES}

[1] M. Tubasihat, S. Madria, "Sensor networks: an overview", IEEE Potentials vol. 22(2), 20-23 (2003)

[2] Schmidt R et al., "Body Area Network BAN, a key infrastructure element for patient-centered medical applications", Biomed Tech (Berl). 2002;47 suppl 1 pt 1:365-8

[3] R. Min, A. Chandrakasan, "Top Five Myths about the Energy Consumption of Wireless Communication", Mobile Computing and Communications Review 2003, 7/1 p. 65-67

[4] A. A. Ahmed, "A Survey on Network Protocols for Wireless Sensor Networks", Information Technology: Research and Education, 2003 proceedings, p. 301-305

[5] M.A.M. Vieira et al., "Survey on wireless sensor network devices", IEEE Emerging Technologies and Factory Automation 2003 proceedings, vol. 1, p. 537-544

[6] E. Mackensen et al., "Smart wireless autonomous microsystems (SWAMs) for sensor actuator networks", ISA/IEEE Sensors for Industry 2004 proceedings, p. 72-78

[7] B.A. Warneke et al., "An autonomous $16 \mathrm{~mm}^{3}$ solarpowered node for distributed wireless sensor networks", IEEE Sensors 2002 proceedings, vol. 2, p. 1510-1515

[8] C. Enz et al., "WiseNET: An Ultralow-Power Wireless Sensor Network Solution", IEEE Computer vol. 37(8), p.62-70

[9] B. Gyselinckx, "System-in-a-cube to improve quality of life", EE Times Communications, August 16, 2004 